\title{
Preparation of Three-Layer Graphene Sheets from Asphaltenes Using a Montmorillonite Template
}

\author{
Xiaolong Wu $\left(D,{ }^{1,2}\right.$ Bojing Ma, ${ }^{2}$ Yisheng $\mathrm{Xu}^{2}{ }^{2}$ Jun $\mathrm{Xu},{ }^{2}$ Haowei Zhai, ${ }^{3}$ Zechen Xiong, \\ Xiangbiao Liao, ${ }^{1}$ Xiaoyang Shi, ${ }^{1}$ and Xi Chen $\mathbb{C}^{1,4}$ \\ ${ }^{1}$ Yonghong Zhang Family Center for Advanced Materials for Energy and Environment, Department of Earth and \\ Environmental Engineering, Columbia University, New York, NY 10027, USA \\ ${ }^{2}$ State Key Laboratory of Chemical Engineering, East China University of Science and Technology, Shanghai 200237, China \\ ${ }^{3}$ Department of Applied Physics and Applied Mathematics, Columbia University, New York, NY 10025, USA \\ ${ }^{4}$ School of Chemical Engineering, Northwest University, Xi'an 710069, China \\ Correspondence should be addressed to Xi Chen; xichen@columbia.edu
}

Received 15 January 2019; Revised 16 May 2019; Accepted 10 June 2019; Published 9 July 2019

Academic Editor: Paulo Cesar Morais

Copyright (c) 2019 Xiaolong Wu et al. This is an open access article distributed under the Creative Commons Attribution License, which permits unrestricted use, distribution, and reproduction in any medium, provided the original work is properly cited.

We propose a novel approach to synthesize graphene sheets with fine quality by calcinating the composites intercalated with montmorillonite (MMT) and asphaltene. The graphene sample thus fabricated could reach $10.79 \mu \mathrm{m}$ long, with smooth and continuous morphology and with little defects. By analyzing the XRD patterns of graphene/MMT composites at different conditions, the graphene formation mechanism is elucidated and the optimal calcination conditions are researched. This study may provide useful insights for low-cost and scalable fabrication of graphene from asphaltene extracted from heavy crude oil.

\section{Introduction}

Since discovered in 2004, graphene has been shown to have prominent physical and electronic properties. Many strategies are developed to prepare graphene, such as oxidationreduction process, and chemical vapor deposition $[1,2]$. However, those methods are high-energy consumptive and the fabrication cost can also be high.

Recently, the fabrication of graphene by converting the low-cost asphalt into carbon nanomaterials provides an alternative approach to take advantage of the byproduct of heavy crude oil [1]. The fundamental aromatic rings of asphaltene can be regarded as fragments of graphene, and the molecular diameter of asphaltene is $1.5 \sim 5.0 \mathrm{~nm}$, which provides an ideal template for graphene formation. Through the calcination process, the aromatic moieties of asphaltene recombine to become graphene.

The 8-10 layers of graphene sheets fabricated from the previous study [1], however, do not testify the prominent proprieties of graphene. A new method needs to be developed so that single-layer graphene or low-layer graphene may be fabricated by limiting the growth of graphene along the vertical direction. Although the synthesis of graphene along the vertical direction remains very hard to control, one could apply a template so that the synthesis takes place within a confined volume. For that matter, the montmorillonite (MMT) emerges as an ideal confinement thanks to its $1 \mathrm{~nm}$ natural interlamellar spacing structure. And the main purpose of this paper is to fabricate large-dimension single- or lowlayer graphene through intercalation with MMT.

In practice, however, the $1 \mathrm{~nm}$ interlamellar spacing may be too limited for the growth of graphene, and modification of the MMT is required. STAB is chosen for MMT modification, through which the lamellar spacing of MMT can be expanded from $1 \mathrm{~nm}$ to $2.1 \mathrm{~nm}[3,4]$, which may provide adequate spacing for asphaltene adsorption. By adjusting different combined calcination conditions including temperature and time, the calcination process is optimized in this work, and large-dimension graphene sheets are prepared by acid treatment. The prepared graphene is examined by XRD, Raman, AFM, SEM, and TEM, and optimum fabrication condition is proposed. The work may inspire low-cost 


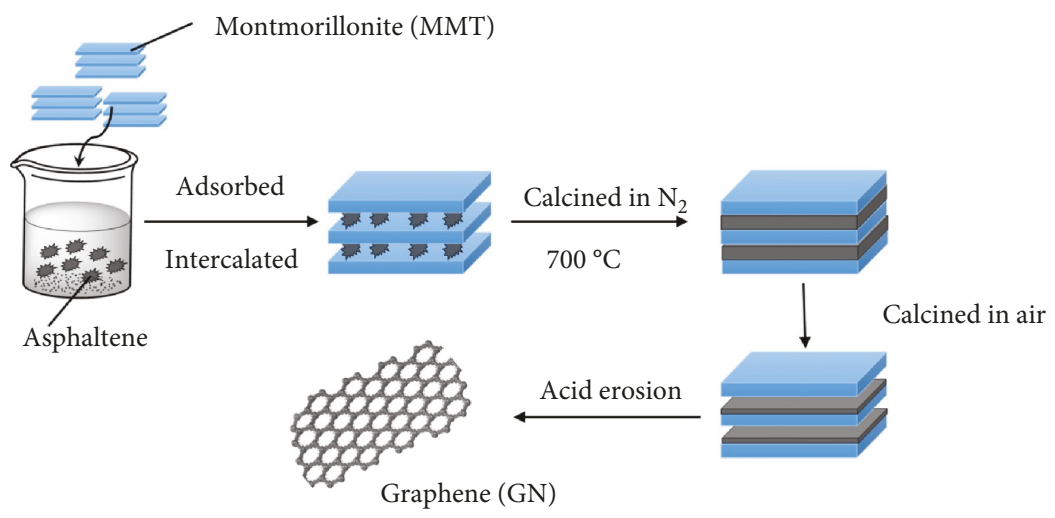

FIgURE 1: Schematic of preparation of large-dimension graphene sheets based on asphaltenes.

and scalable production of graphene through engineered MMT and calcination process.

\section{Experimental}

2.1. Materials. Asphaltenes are extracted from Tahe heavy crude oil. MMT is pretreated following the procedure reported elsewhere [3,5]: $50.0 \mathrm{~g}$ MMT is mixed with $4.0 \mathrm{~g}$ $\mathrm{Na}_{2} \mathrm{CO}_{3}$ and stirred for 30 min every $3 \mathrm{~h}$ for $24 \mathrm{~h}$. After that, MMT is filtrated by suction filtration from the solution and dried in an oven at $80^{\circ} \mathrm{C}$ in vacuum for $6 \mathrm{~h}$. Next, 3.0 CEC STAB is fed slowly into 5.0 wt.\% MMT solution under stir at $80^{\circ} \mathrm{C}$ for $4 \mathrm{~h}$, followed by filtration and washing until no $\mathrm{Br}^{-}$is detected. Finally, the modified MMT is grinded and then sieved by a 325 -mesh sieve.

2.2. Preparation of Graphene. The procedure for graphene synthesis is illustrated in Figure 1. Firstly, $1.0 \mathrm{~g}$ asphaltene and $20 \mathrm{ml}$ toluene are mixed and subjected to ultrasonication for $1 \mathrm{~h}$. Next, $10.0 \mathrm{~g}$ treated MMT is slowly fed in and stirred for $1 \mathrm{~h}$. Asphaltene/MMT composites are collected after filtration and dried at $100^{\circ} \mathrm{C}$ in vacuum. The composite is then pyrolyzed in $\mathrm{N}_{2}$ for $2 \mathrm{~h}$ at $700^{\circ} \mathrm{C}$ [1] and calcined in air for $15 \mathrm{~min}$ at $700^{\circ} \mathrm{C}$ in muffle. During this step, graphene is synthesized between the layers of MMT. Finally, the graphene/MMT composite is etched in the solution of $10 \mathrm{wt} . \% \mathrm{HF}$ and $10 \mathrm{wt} . \% \mathrm{HCl}$ under stirring. The graphene is filtrated and dried until the color of filtrate became transparent. After acid wash, the absolute asphaltene could be scooped up from the supernatant liquid.

\section{Results and Discussion}

3.1. Structural Characterization of Graphene. X-ray power diffraction (XRD; Bruker) is used to examine the structure of graphene [6]. Samples prepared from asphaltene/MMT composites calcined in air with different conditions are examined, so that an optimal process may be identified. Figures 2(c) and 2(d) show the comparison of XRD diffraction patterns of GN/MMT samples calcined in air at different times ranging from $15 \mathrm{~min}$ to $2 \mathrm{~h}$, at $600^{\circ} \mathrm{C}$ and $700^{\circ} \mathrm{C}$, respectively. According to the Bragg equation $(2 d \cdot \sin \theta=n \cdot \lambda)$, the characteristic peak at $2 \theta=23.15^{\circ}$ corresponds to the interpla- nar spacing value of $0.38 \mathrm{~nm}$. The characteristic peak at $2 \theta$ $=23.15^{\circ}$ exists in all conditions except for $2 \mathrm{~h}$ calcinations regardless of the temperature used.

In Figure 2(a), the weak peak at around $23.1^{\circ}$ is nearly 0 , which indicates that there is no characteristic peak similar to graphene. The weak peaks at around $23.1^{\circ}$ for $2 \mathrm{~h}$ calcination at both $600^{\circ} \mathrm{C}$ and $700^{\circ} \mathrm{C}$ (Figures $2(\mathrm{c})$ and $2(\mathrm{~d})$ ) indicate that most of the graphene are burnt out, and the montmorillonite is decomposed under such high temperature so that there are no characteristic peaks of montmorillonite in Figures 2(c) and 2(d). For other calcination conditions, the detailed information including peak height, full-width at half-maximum (FWHM), and crystallinity of GN/MMT in XRD patterns is presented in Table 1 . The crystallinity is calculated by using MDI Jade 6. The higher value of peak height in XRD indicates the larger amount. The results indicate that more graphene with lower crystallinity is obtained at $600^{\circ} \mathrm{C}$, while the sample with the highest crystallinity is obtained at $700^{\circ} \mathrm{C}$.

Owing to the high crystallinity being positively correlated with fine quality, the condition of $15 \mathrm{~min}$ calcination at $700^{\circ} \mathrm{C}$ is regarded as the optimal calcination condition in the present study. The purified graphene is obtained after acid erosion and wash. The XRD pattern of the purified graphene at $23.09^{\circ}$ (Figure 2(b)) shows that purification improves the crystallinity of graphene to $81.90 \%$ at the optimal condition mentioned above. In addition, the peaks around $15^{\circ} \sim 17^{\circ}$ indicate the existence of some unconfirmed impurities.

Raman spectroscopy is useful for confirming the presence of $\mathrm{sp}^{2}$ phase in graphene [7], and in Figure 2(e), two typical bands are shown at $1596 \mathrm{~cm}^{-1}$ ( $\mathrm{G}$ band) corresponding to the $\mathrm{E}_{2 \mathrm{~g}}$ phonon at the Brillouin zone and at $1346 \mathrm{~cm}^{-1}$ (D band), indicating a disordered structure or the edge of the sample associated with structural defects $[2,8-12]$. The intensity of $\mathrm{D}$ band is 125 , and the intensity of $\mathrm{G}$ band is 152.5 , thus the $I_{\mathrm{D}} / I_{\mathrm{G}}$ intensity ratio $(0.82)$ is much smaller than the graphene similarly prepared from asphaltene by $\mathrm{Xu}$ et al. [1], reflecting better quality with fewer vacancies or disorder defects [13]. Since the adsorption spacing of modified MMT is just about half of the spacing of modified vermiculite [1], it provides more appropriate spacing for graphene synthesis and facilitates the fabrication of low-layer graphene sheets. Owing to the smaller spacing for graphene synthesis, the pyrolysis time in $\mathrm{N}_{2}$ can be increased from 


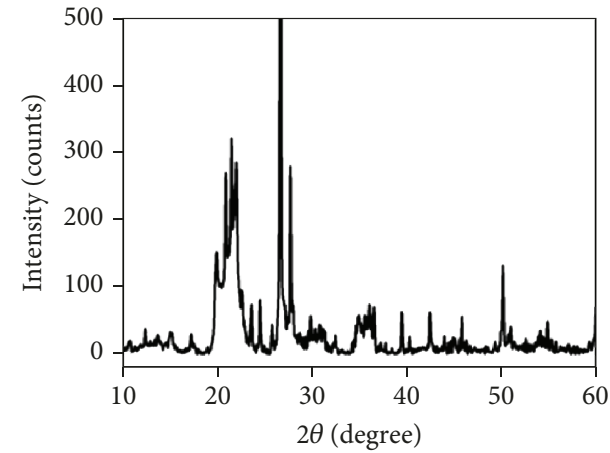

(a)

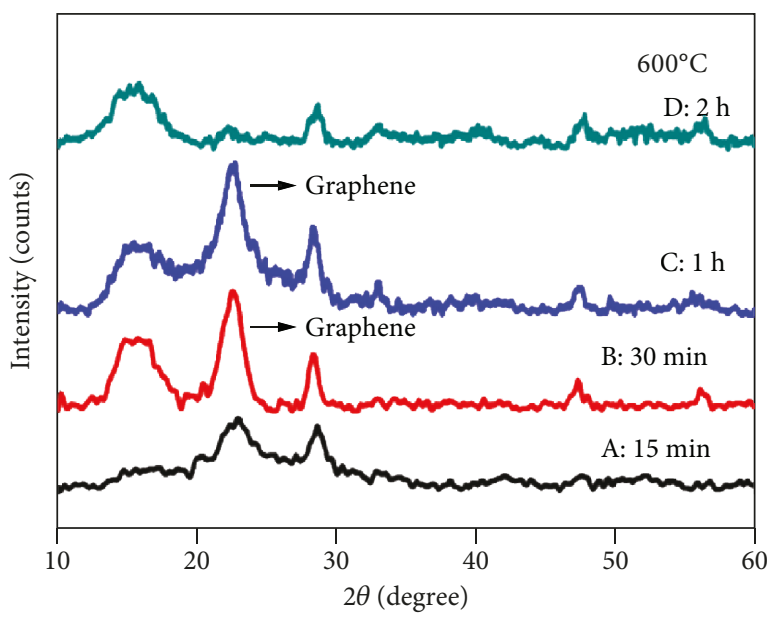

(c)

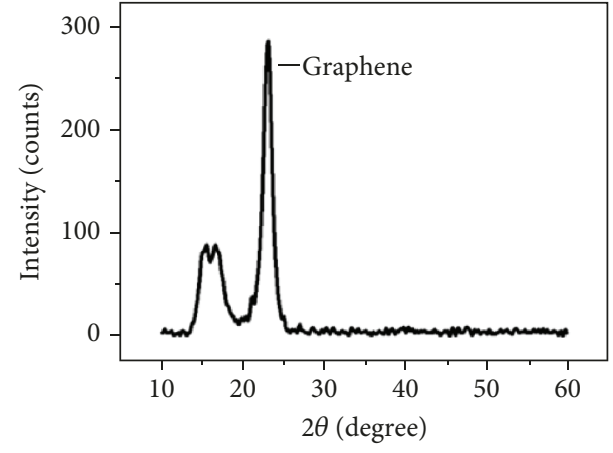

(b)

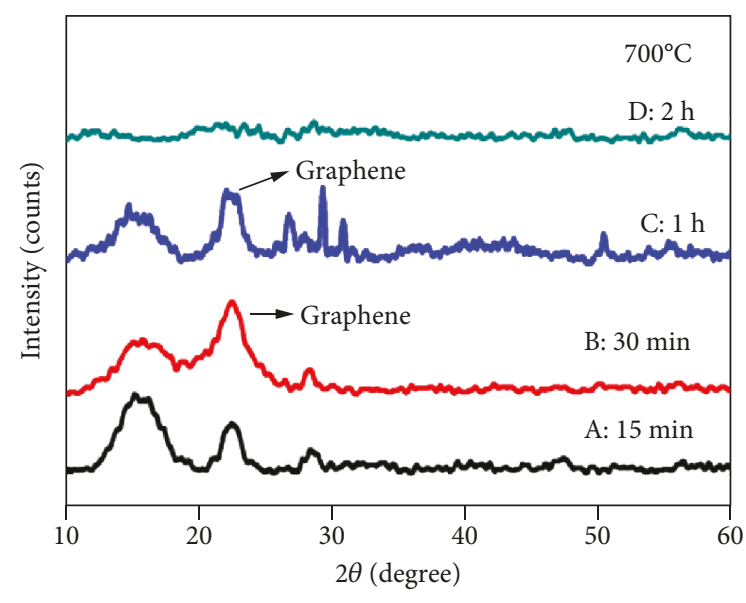

(d)

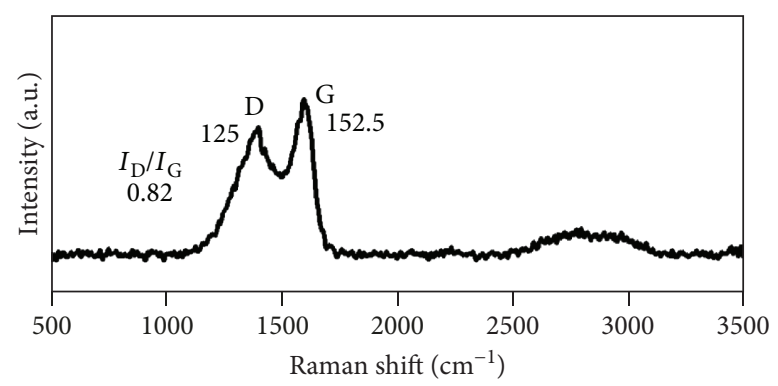

(e)

FIGURE 2: XRD patterns of modified montmorillonite (a) and purified graphene sheets (b) and different calcining time of graphene/MMT samples at $600^{\circ} \mathrm{C}(\mathrm{c})$ and $700^{\circ} \mathrm{C}(\mathrm{d})$ without erosion treatment and the Raman spectrum (e) of the prepared graphene with $I_{\mathrm{D}} / I_{\mathrm{G}} 0.82$ at around $23.1^{\circ}$.

TABLE 1: Results of XRD analysis of graphene/MMT samples at six conditions.

\begin{tabular}{lccccc}
\hline Temperature & $\begin{array}{c}\text { Time } \\
(\min )\end{array}$ & $2 \theta\left(^{\circ}\right)$ & $\begin{array}{c}\text { Peak } \\
\text { height }\end{array}$ & $\begin{array}{c}\text { FWHM } \\
\left({ }^{\circ}\right)\end{array}$ & $\begin{array}{c}\text { Crystallinity } \\
(\%)\end{array}$ \\
\hline \multirow{3}{*}{$600^{\circ} \mathrm{C}$} & 15 & 23.11 & 21 & 2.317 & 50.84 \\
& 30 & 23.09 & 97 & 1.913 & 45.19 \\
& 60 & 23.12 & 97 & 2.449 & 54.43 \\
$700^{\circ} \mathrm{C}$ & 15 & 23.09 & 33 & 1.620 & 78.63 \\
& 30 & 22.92 & 67 & 2.635 & 33.93 \\
& 60 & 23.12 & 33 & 1.762 & 59.90 \\
\hline
\end{tabular}

$1 \mathrm{~h}$ to $2 \mathrm{~h}$ to ensure the adequate carbonization of asphaltene molecules intercalated into MMT, and the $I_{\mathrm{D}} / I_{\mathrm{G}}$ intensity ratio is reduced consequently. And the step of calcination in air removes the allotropes of graphene. These results are consistent with the high crystallinity without allotropes from $\mathrm{XRD}$ analysis.

3.2. Topographical Analysis of Graphene. Graphene sheet prepared under the condition of $700^{\circ} \mathrm{C}$ for $15 \mathrm{~min}$ is under investigation in the section. The topography and the thickness of prepared graphene are examined by atomic force microscope (AFM; Veeco). Figure 3(a) shows that the graphene is coherent with little defects, attaining a length of 


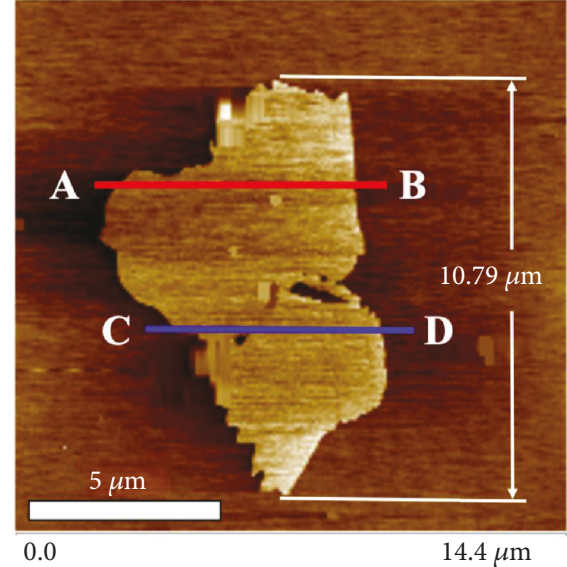

(a)

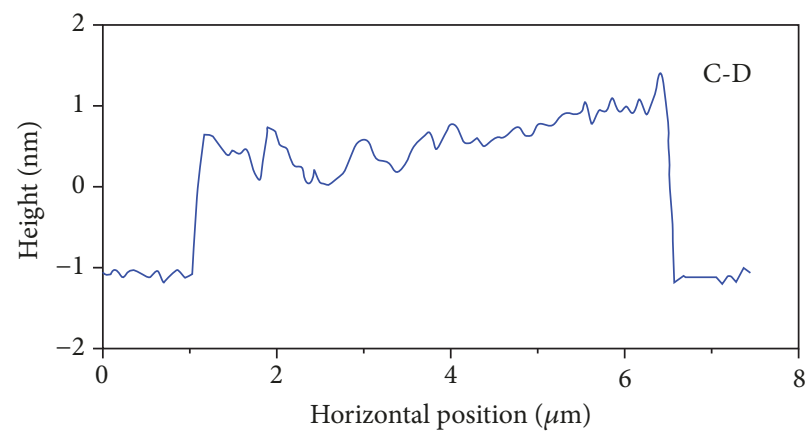

(c)

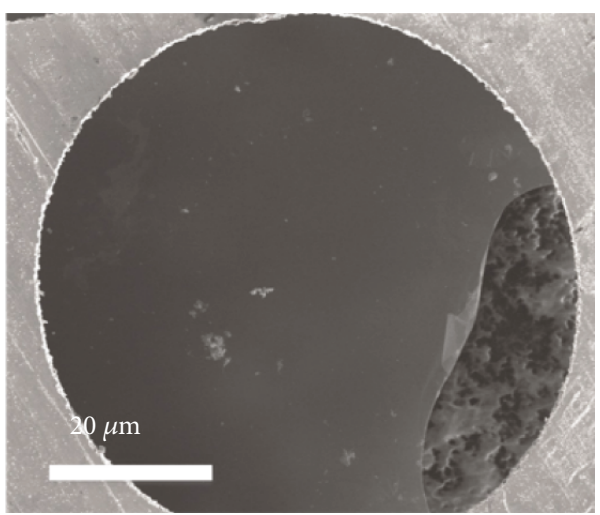

(e)

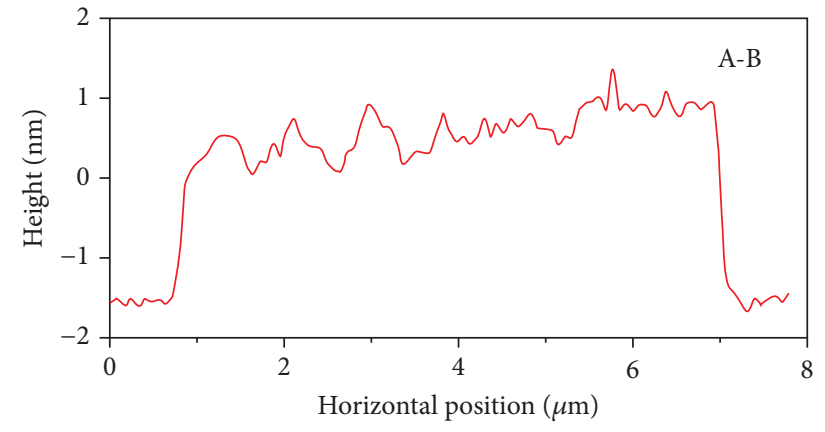

(b)

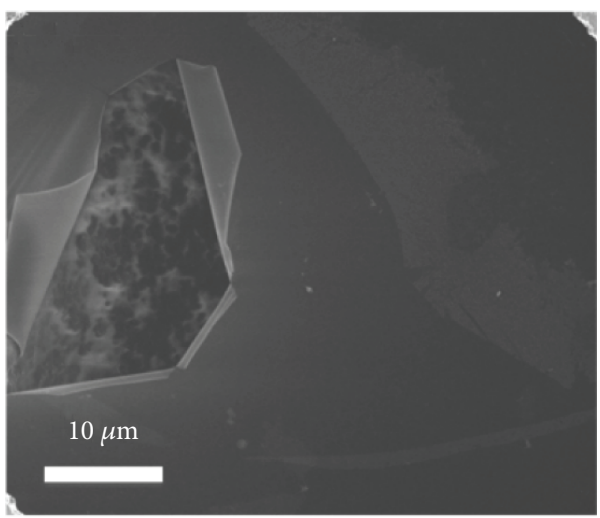

(d)

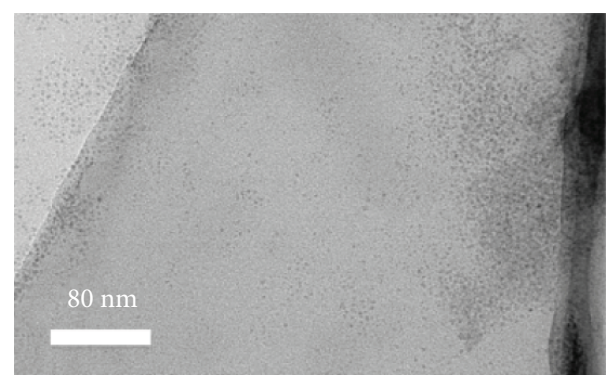

(f)

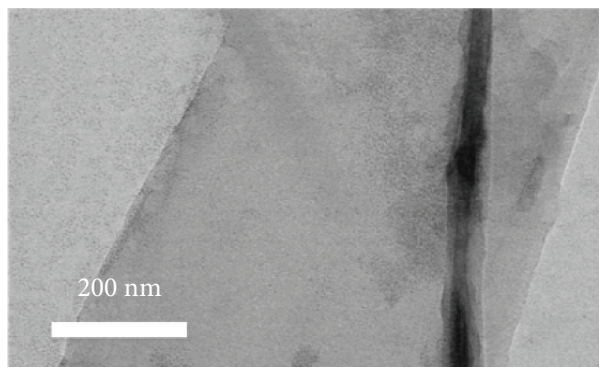

(g)

Figure 3: AFM image (a) and height profiles $(b, c)$ of the asphaltene-derived graphene sheet. SEM (d, e) and TEM (f, g) images of the asphaltene-derived graphene sheets. 
$10.79 \mu \mathrm{m}$ and with a width of $6 \sim 7 \mu \mathrm{m}$. AFM analysis (Figures 3(b) and 3(c), along lines $\mathrm{AB}$ and $\mathrm{CD}$, respectively) indicates a flat feature with a consistent thickness of $\sim 1.81 \mathrm{~nm}$. Since the thickness of ideal single-layer graphene is approximately $0.34 \mathrm{~nm}$ [14], the present graphene sheet may represent three layers of graphene according to the three-layer graphene with height $\sim 1.9 \mathrm{~nm}$ by Li et al. [15]. Furthermore, the RMS (root mean square) roughness estimated with AFM data is $0.31 \mathrm{~nm}$ over a $60 \mu \mathrm{m}^{2}$ area [16], reflecting an overall smooth surface of the graphene plane [17].

The morphology of the graphene samples is investigated using a scanning electron microscope (SEM; FEI NOVA Nano SEM 450) and high-resolution transmission electron microscopy (HRTEM; JEOL JEM-2100). Figures 3(d) and 3(e) show the scanning image of asphalt-derived graphene sheet attached to a copper sheet. The graphene sheet is quite smooth with little defects. In addition, a graphene sheet with the size up to $600 \mu \mathrm{m}$ can be obtained by using the current method. According to the HRTEM images (Figures 3(f) and $3(\mathrm{~g})$ ), the asphaltene-derived graphene sheet is fairly smooth and transparent, which is in close agreement with the observations in AFM.

According to relevant studies [18-20], double-layered graphene sheet may become very attractive. To obtain even fewer layers of graphene, the MMT may be modified from hydrophilicity to lipophilicity without the expansion of interlamellar spacing of MMT, so that double-layered graphene might be possibly generated within the interlamellar spacing of around $1.5 \mathrm{~nm}$. Moreover, recarbonization may lead to a larger scale of graphene sheets. These studies will be carried out in the future.

\section{Conclusions}

A novel low-cost approach to prepare low-layer graphene is presented by intercalation of asphaltene and MMT. Thanks to the appropriate interlamellar spacing provided by modified MMT, which blocks the crispation of asphaltene molecules to forming carbon nanotubes but only graphene sheets. The intercalation degree of asphaltene by thermogravimetric analysis (TGA) and recycle of unreacted asphaltene should be investigated in future works to get the optimal strategy to maximize the yield. The longer pyrolysis time in $\mathrm{N}_{2}$ atmosphere reduces the $I_{\mathrm{D}} / I_{\mathrm{G}}$ intensity ratio and defect, which improves the quality of the obtained graphene sheets. In addition, the calcination process in air removes the allotropes of graphene to approach high crystallinity. By using low-cost raw chemicals from the heavy oil and calcined at optimal conditions $\left(15 \mathrm{~min}\right.$ at $\left.700^{\circ} \mathrm{C}\right)$, three-layer graphene sheets with large dimension and high quality (with high crystallinity and low defect ratio) are successfully obtained. Microscopy examination confirmed the integrity of the prepared graphene. The proposed methodology has the advantage over other chemical pathways, such as oxidationreduction, which may preserve the pristine structure and maintain the integrity of graphene. The present work may inspire further study of scalable production of low-layer graphene sheets.

\section{Data Availability}

The data used to support the findings of this study are included within the article.

\section{Conflicts of Interest}

There is no conflict of interests in this article.

\section{Acknowledgments}

Financial support by the National Natural Science Foundation of China (51003028, 21004021, 21306049, and 51273063), the Fundamental Research Funds for the Central Universities, the Higher School Specialized Research Fund for the Doctoral Program (20110074110003), and the 111 Project grant (B08021) is gratefully acknowledged. The authors also thank Petrochina Liaohe Oilfield Company for yielding oil samples and technological supports.

\section{References}

[1] C. Xu, G. Ning, X. Zhu et al., "Synthesis of graphene from asphaltene molecules adsorbed on vermiculite layers," Carbon, vol. 62, pp. 213-221, 2013.

[2] Z. Tu, Z. Liu, Y. Li et al., "Controllable growth of 1-7 layers of graphene by chemical vapour deposition," Carbon, vol. 73, pp. 252-258, 2014.

[3] H. A. Essawy, A. S. Badran, A. M. Youssef, and A. E. F. A. Abd el-Hakim, "Polystyrene/montmorillonite nanocomposites prepared by in situ intercalative polymerization: influence of the surfactant type," Macromolecular Chemistry and Physics, vol. 205, no. 17, pp. 2366-2370, 2004.

[4] L. Wang and A. Wang, "Adsorption properties of Congo red from aqueous solution onto surfactant-modified montmorillonite," Journal of Hazardous Materials, vol. 160, no. 1, pp. 173-180, 2008.

[5] X. Feng, G. Hu, X. Meng, Y. Ding, S. Zhang, and M. Yang, "Influence of ethanol addition on the modification of montmorillonite by hexadecyl trimethylammonium bromide," Applied Clay Science, vol. 45, no. 4, pp. 239-243, 2009.

[6] R. K. Jammula, A. Tumuluri, N. K. Rotte, K. C. James Raju, and V. V. S. S. Srikanth, "Cupric oxide decked few-layered graphene: synthesis and dielectric behaviour," Carbon, vol. 78, pp. 374-383, 2014.

[7] M. A. Pimenta, G. Dresselhaus, M. S. Dresselhaus, L. G. Cançado, A. Jorio, and R. Saito, "Studying disorder in graphitebased systems by Raman spectroscopy," Physical Chemistry Chemical Physics, vol. 9, no. 11, pp. 1276-1290, 2007.

[8] S. M. Clark, K. J. Jeon, J. Y. Chen, and C. S. Yoo, "Few-layer graphene under high pressure: Raman and X-ray diffraction studies," Solid State Communications, vol. 154, pp. 15-18, 2013.

[9] L. M. Malard, M. A. Pimenta, G. Dresselhaus, and M. S. Dresselhaus, "Raman spectroscopy in graphene," Physics Reports, vol. 473, no. 5-6, pp. 51-87, 2009.

[10] L. G. Cançado, A. Jorio, E. H. M. Ferreira et al., "Quantifying defects in graphene via Raman spectroscopy at different excitation energies," Nano Letters, vol. 11, no. 8, pp. 3190-3196, 2011. 
[11] S. Lu, M. Yao, X. Yang et al., "High pressure transformation of graphene nanoplates: a Raman study," Chemical Physics Letters, vol. 585, pp. 101-106, 2013.

[12] M. J. Allen, V. C. Tung, and R. B. Kaner, "Honeycomb carbon: a review of graphene," Chemical Reviews, vol. 110, no. 1, pp. 132-145, 2010.

[13] A. Eckmann, A. Felten, A. Mishchenko et al., "Probing the nature of defects in graphene by Raman spectroscopy," Nano Letters, vol. 12, no. 8, pp. 3925-3930, 2012.

[14] X. Li, J. Li, X. Zhou et al., "Silver nanoparticles protected by monolayer graphene as a stabilized substrate for surface enhanced Raman spectroscopy," Carbon, vol. 66, pp. 713$719,2014$.

[15] X. Li, X. Wang, L. Zhang, S. Lee, and H. Dai, "Chemically derived, ultrasmooth graphene nanoribbon semiconductors," Science, vol. 319, no. 5867, pp. 1229-1232, 2008.

[16] Y. Yao and C.-p. Wong, "Monolayer graphene growth using additional etching process in atmospheric pressure chemical vapor deposition," Carbon, vol. 50, no. 14, pp. 5203-5209, 2012.

[17] I. F. Cheng, Y. Xie, R. Allen Gonzales et al., "Synthesis of graphene paper from pyrolyzed asphalt," Carbon, vol. 49, no. 8, pp. 2852-2861, 2011.

[18] X. Fan, K. Elgammal, A. D. Smith et al., "Humidity and $\mathrm{CO}_{2}$ gas sensing properties of double-layer graphene," Carbon, vol. 127, pp. 576-587, 2018.

[19] M. Liu, X. Yin, and X. Zhang, "Double-layer graphene optical modulator," Nano Letters, vol. 12, no. 3, pp. 1482-1485, 2012.

[20] L. Thirugnanam and R. Sundara, "Few layer graphene wrapped mixed phase $\mathrm{TiO}_{2}$ nanofiber as a potential electrode material for high performance supercapacitor applications," Applied Surface Science, vol. 444, pp. 414-422, 2018. 


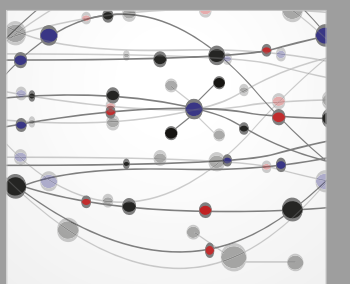

The Scientific World Journal
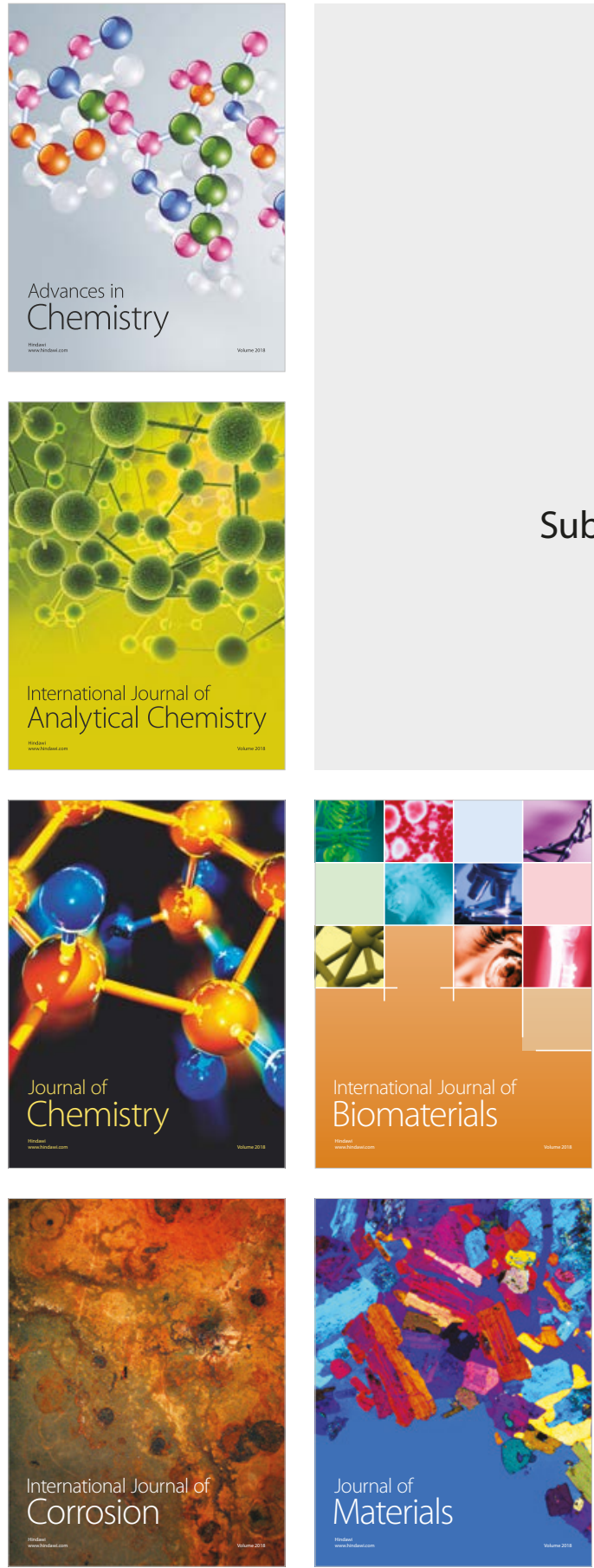

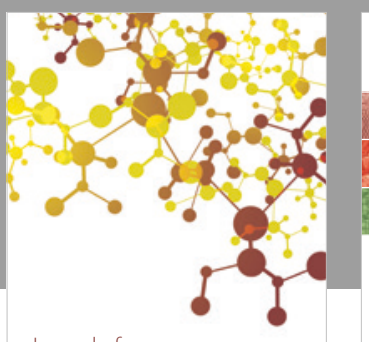

Journal of

Applied Chemistry
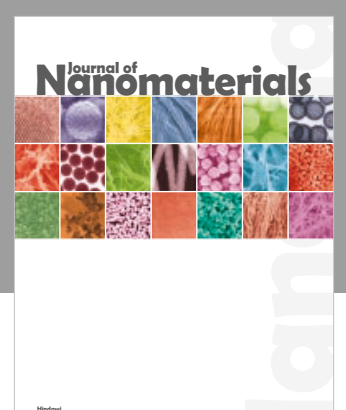

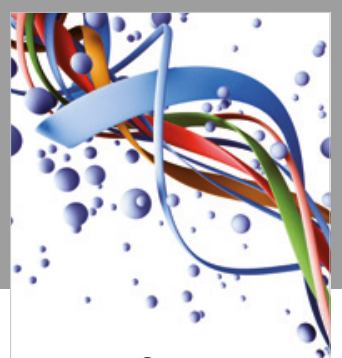

Scientifica

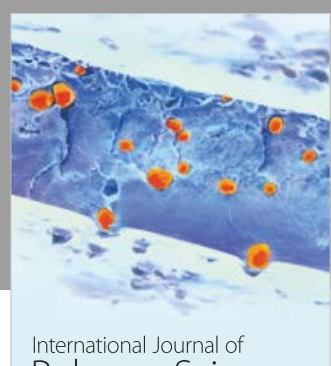

Polymer Science

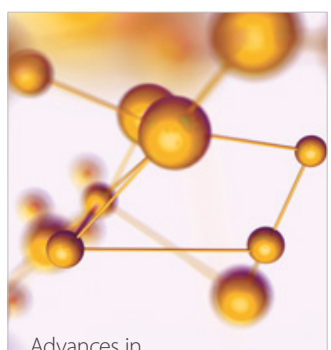

Physical Chemistry
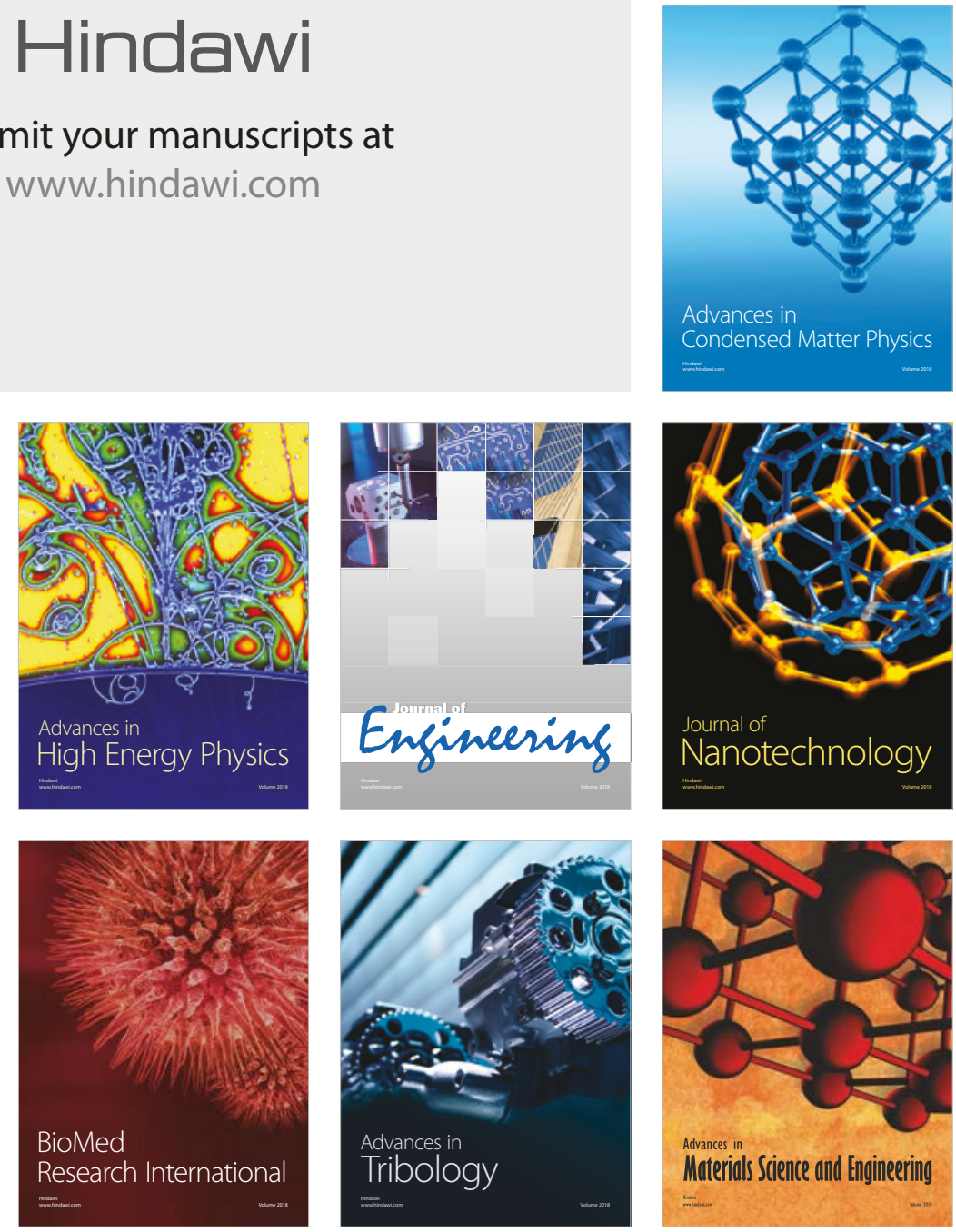